\title{
Development of Experimental Prototypes for the Teaching of Science
}

\author{
Guillermo Terán ${ }^{1}$, Fausto Ávalos ${ }^{2}$, Washington Lomas ${ }^{3, *}$ and Iliana Quintana ${ }^{3}$ \\ ${ }^{1}$ Universidad Central del Ecuador, Faculty of Filosofía, Letras y Ciencias de la Educación, EC-170521, Quito, Ecuador \\ ${ }^{2}$ Universidad Central del Ecuador, Faculty of Ingeniería y Ciencias Aplicadas, EC-170521, Quito, Ecuador \\ ${ }^{3}$ Universidad Central del Ecuador, Centro de Física, EC-170521, Quito, Ecuador
}

\begin{abstract}
The following document presents the development of educational experimental prototypes. The themes of the prototypes cover principles or concepts of physics. Like kinematics, oscillatory movements and shocks. Each prototype has sensors that allow measurements of the variables involved in the experimentation. At the same time, mobile applications are developed for devices with Android operating system, which allow the user to control the prototype from their cell phone and also view the variables measured by the experimentation prototypes. The applications also have a data export system that allows to obtain a file in csv format. In this way, the measurements are obtained in the form of a table to later be processed through office automation tools or other numerical computing software such as Matlab.
\end{abstract}

\section{Introduction}

To improve the learning experience of the students of the Universidad Central del Ecuador and in order to reinforce their knowledge through experimentation, experimental prototypes are developed which, through the use of technology, allow students to carry out practices that demonstrate physical principles or concepts.

The interaction with the laboratory prototypes will have a positive impact on the creativity and development of cognitive thinking of the students since the transfer of knowledge with acquisition and interpretation of data will be efficient, guaranteeing the consolidation of concepts of the different themes of Physics such as a branch of the Sciences.

\section{Methodology}

Starting from physical principles, variouscriteria are analyzed for the development ofexperimental prototypes. In this first stage, topics such as parabolic movement, shocks, capacitors, light composition have been chosen.

Each prototype consists of:

- Experimentation equipment: includes all the necessary materials to carry out the experimentation, within each of them there is an electronic system which contains one or more sensors necessary to carry out the measurements of variables that allow the completion of the experiment, a communication system that allows the information obtained to be sent to the mobile device and allows the user to interact with the equipment.
- Mobile application for devices with Android operating system: each experimentation equipment has a mobile application, which allows the user to manipulate the equipment and observe the measurements made. Depending on each experiment there are different options within the application, within which graphs of each of the variables measured with respect to time can be observed.

Below, the components of each prototype mentioned above are detailed in a better way.

\subsection{Parabolic Movement.}

Inside the prototype of the parabolic movement there is a system actuated by a spring, which allows a particle to be thrown, in this case a small sphere to carry out the experimentation. The launch angle is configured through the mobile application, by means of a servomotor. In the cannon we have a pair of infrared sensors that allow us to measure the initial velocity of the particle. Additionally, there is a small platform that has an impact sensor which allows obtaining the time of flight of the particle.

For experimentation purposes, the friction between the sphere and the barrel is considered to be zero.

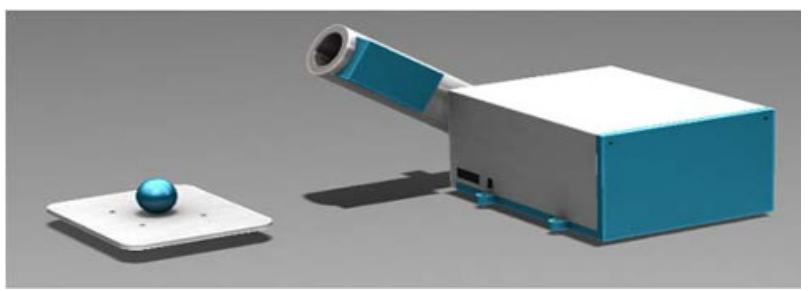

Fig. 1. Parabolic movement experimentation prototype. 
The mobile application that allows interaction with the prototype is shown in Fig.

There are the following options within the application, among which we have:

- Connect button: allows you to select the bluetooth device with which to connect, in this case with the parabolic movement experimentation prototype.

- Disconnect button: allows you to disconnect the experimentation prototype.

- The status label shows if the prototype is connected or disconnected.

- $\quad$ Through the slider the launch angle of the particle is selected and once selected, the angle is configured through the OK button.The initial speed measured by the prototype is shown on the "Initial Speed" label.

- - The "Flight Time" label shows the flight time measured by the prototype.

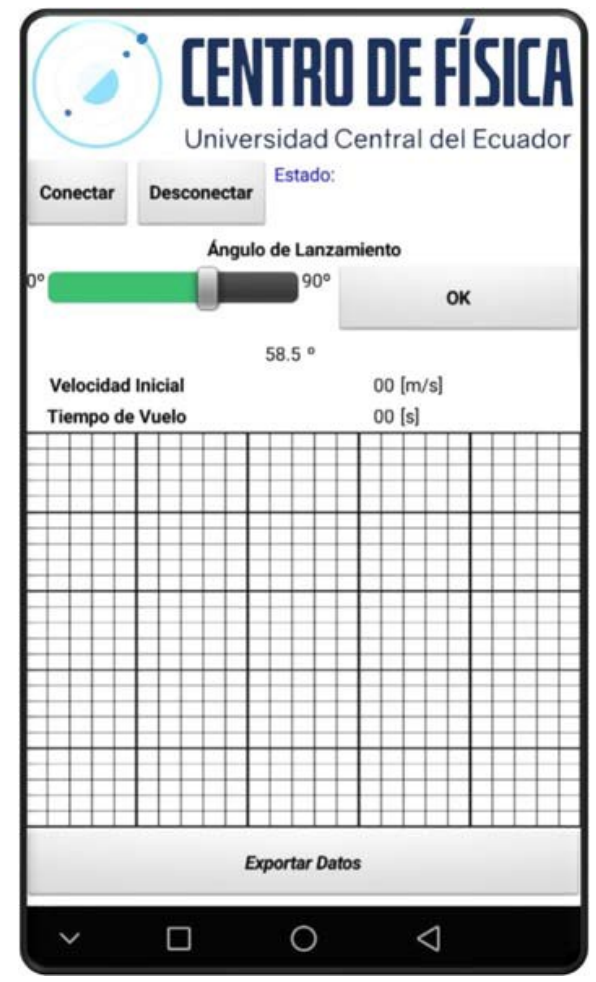

Fig. 2. Mobile application of the parabolic movement prototype.

\subsection{One-dimensional shocks.}

In the prototype of the one-dimensional collision, there is a system activated by a spring, which allows a particle to be thrown, which impacts with another sphere that is in the line of action of the first sphere. In this way both spheres impact, and through sensors placed at the initial end and at the end end, the initial conditions before the crash and the final conditions after the crash can be obtained. The sensors placed are a pair of infrared sensors at the initial end and at the end end to thereby measure the velocities of the particles before and after the collision. For the purposes of experimentation, it is considered that the friction between the test spheres and the track on which the experiment is carried out is zero.

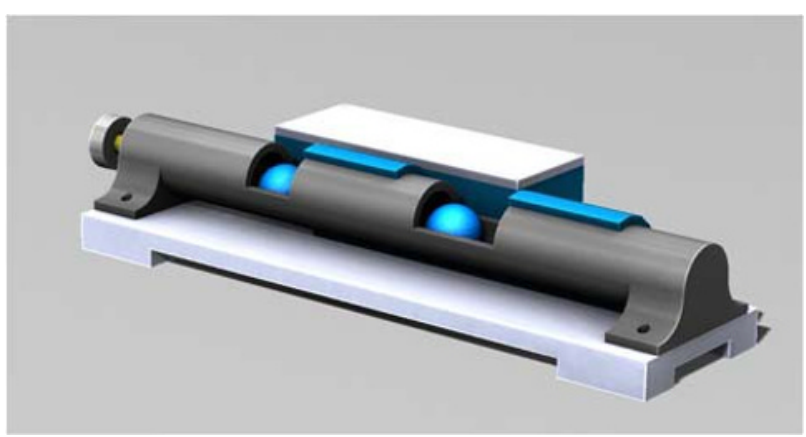

Fig. 3. One-dimensional shocks experimentation prototype

In Fig. 4 the application is shown to be able to interact with the prototype. Similar to the application described above, there are buttons, for example the prototype connection and disconnection buttons. In addition to the label that indicates if the prototype is connected or disconnected. This application additionally has:

- Two labels in which the initial conditions before the collision are observed, that is, the initial speeds. For the particle that is impacted in this case, a null initial velocity will be considered.

- Two labels showing the conditions after the crash, speeds after the crash.

- In case of placing spheres of different mass, there are two text boxes in which the masses of each of the spheres can be entered.

- In addition, it has three additional labels in which the kinetic energy is shown before and after the crash. It iscalculated from the velocity data and mass data of the test spheres.

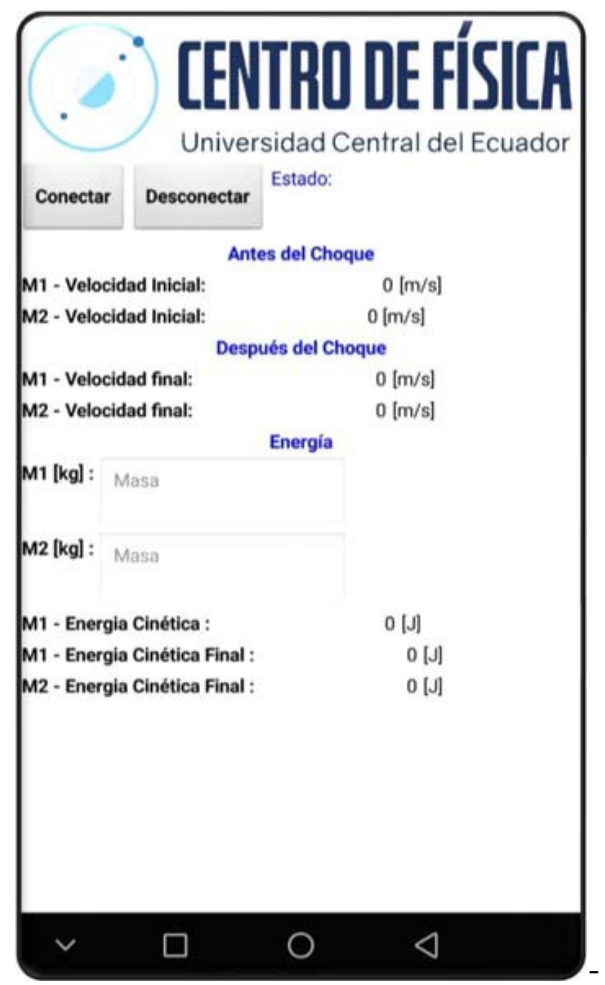

Fig. 4. One-dimensional shocks prototype mobile application 


\subsection{Charging and Discharging Capacitors.}

The prototype developed for charging and discharging capacitors is shown in Fig. 5. It allows observing the charge and discharge graphs of an R-C set in series. Resistance and capacitance values can be changed as user substitutes. In the upper part it has a switch which allows you to choose if you want tocharge the capacitor or discharge it. In the application, the voltage-time graph is observed to see how the voltage changes according to the R-C set placed.

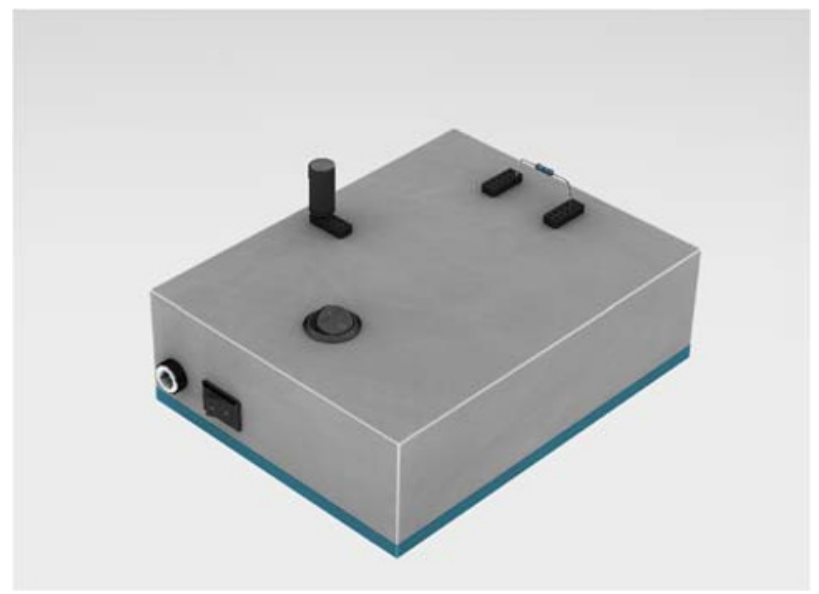

Fig. 5. Capacitor charging and discharging experimentation prototype

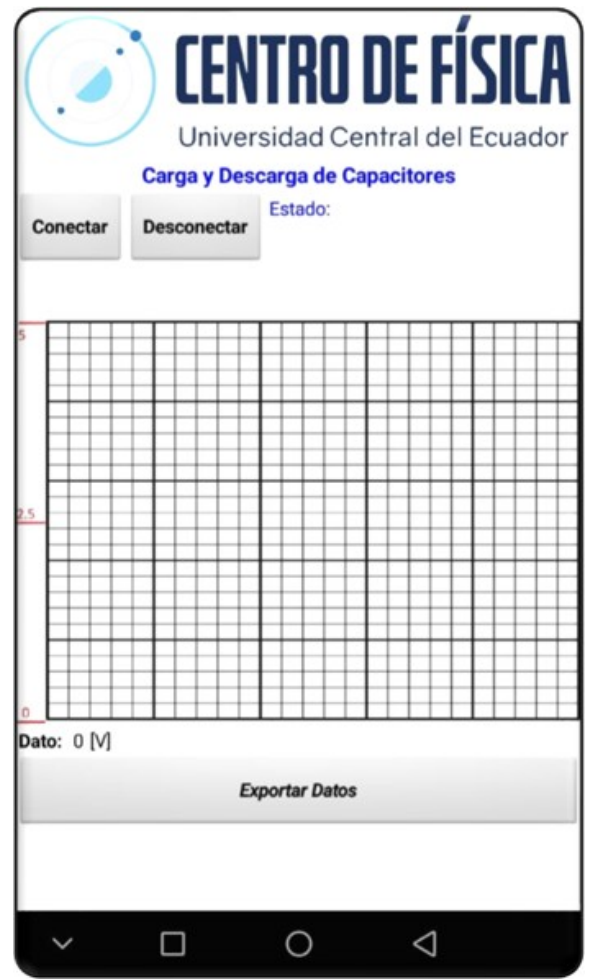

Fig. 6. Capacitor charging and discharging prototype mobile application

In Fig. 6 you can see the application developed for the prototype, the connection and disconnection buttons as well as the connection status label is like the previous ones described. If you have a squared area which allows you to see the graph of voltage - time of the charge or discharge process. In this case, the prototype uses a charging voltage of $5 \mathrm{~V}$. In addition, there is a label that allows you to see what the instantaneous voltage is.

The voltage-time data is recorded and can be exported to obtain a file in csv format. To obtain the file with the registered data, use the Export Data button.

\subsection{Composition of light.}

The following prototype allows us to observe the additive synthesis of colors. Different colors are obtained from a set of RGB LEDs. The prototype is shown in Fig. 7.

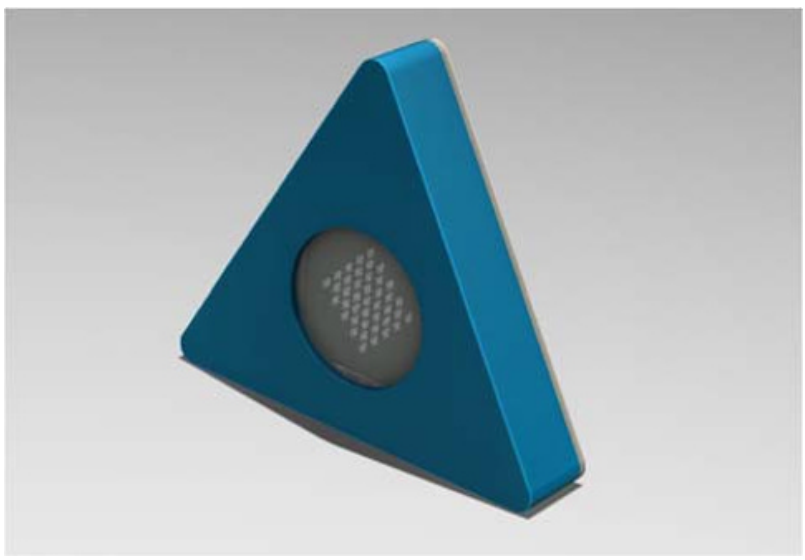

Fig. 7. Light composition experimentation prototype

The application that allows selecting the prototype light is seen in Fig. 8. The connection and disconnection buttons as well as the connection status label were previously detailed.

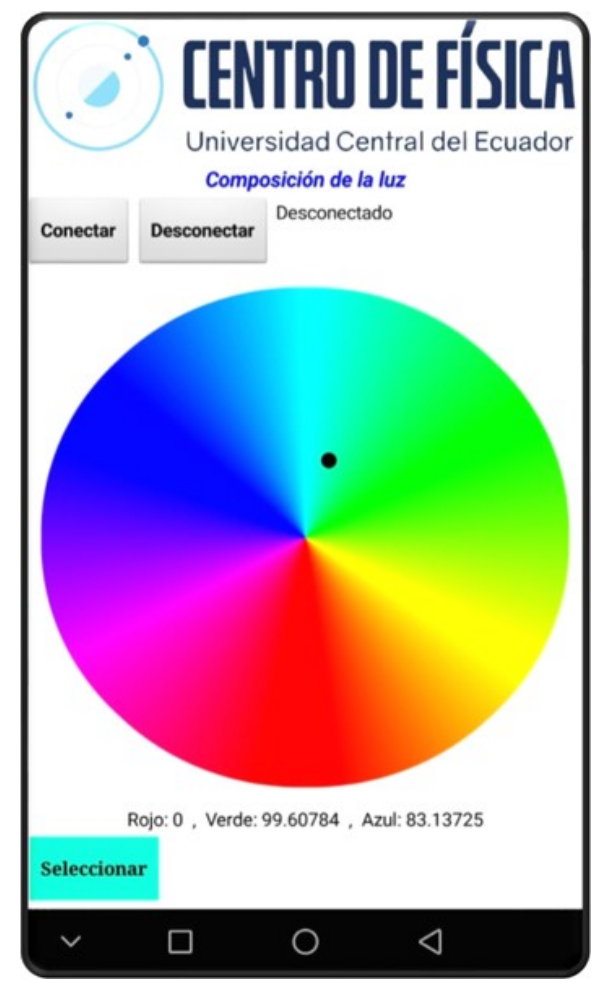

Fig. 8. Light Composition Prototype Mobile App 
There is a wheel-shaped color palette from which you can select a color from it. Below the color wheel, you can see the composition percentage of each selected color: red, green and blue. Once the color has been selected tobe reflected in the prototype, the "Select" button must be selected.

\section{Results}

Each of the developed prototypes allows obtaining data which can be compared with data obtained from the respective theory.

Inside each of the prototypes there is a bluetooth module which was renamed so that it can be quickly identified within the pairing with the Android device.

\subsection{Parabolic Movement.}

The parabolic movement prototype is in operation, allowing the initial speed and time of flight of the particle to be obtained.

The application correctly shows the data obtained, for the prototype test an Arduino Nano development board is being used. At the end of the first tests, a printed circuit board of the prototype will be used, after this process the prototype will be next to go to tests and validation by the work team of the Centro de Física de la Universidad Central del Ecuador.

\subsection{One-dimensional shocks.}

When carrying out the experimentation, it is observed that sometimes the spheres bounce off the walls of the cylindrical track within which the experimental process is carried out. Because of this, experimentation is done a couple of times to get the data without these disturbances.

At the moment an Arduino development board is being used for prototype testing, the next step is to only use the microcontroller inside a printed circuit board to decrease the size of the components used. Once this process has been carried out, the prototype will go through tests and validation by the team of the Centro de Física de la Universidad Central del Ecuador.

\subsection{Charging and Discharging Capacitors.}

The prototype is in operation, thanks to the small graphic area of the application, it is possible to observe the voltage - time graphs, allowing to observe the charge and discharge graphs of the capacitor in the $\mathrm{R}-\mathrm{C}$ assembly, similar to a small oscilloscope.

Knowing the time constant of an $\mathrm{R}-\mathrm{C}$ series circuit (resistance - Capacitor), the exported data representing the voltage - time values can be used to calculate the capacitance values with which it was used for experimentation. Obtaining errors of $3 \%$.
This prototype is demonstrative, the selected color can be obtained through the mobile application. In the back it has holes which allow air circulation to reduce the temperature of the transistors used as drivers.

\section{Conclusion}

- Each prototype allows the measurement of the minimum variables to obtain a comparison with the theoretical data obtained, in this way the experimentation with the theory is associated.

- The interface of each application is friendly to the user, showing the data in a clear way. Each application has common elements which will allow students to quickly adapt to each of the prototypes.

- The use of applications makes the student show a greater interest in the experimentation and study of Physics.

- The percentage of error of the data obtained compared with the data of the respective theory is less than $5 \%$.

- The prototypes are low cost, using materials easily accessible in the Ecuadorian market.

\section{References}

1. R.A. Serway, J.W. Jewitt, Física para ciencias e ingeniería, 10, (2019)

2. F.P. Beer, E.R. Johnston, P.J. Cornwell, Mecánica Vectorial para ingenieros Dinámica, 9, (2010)

3. H. Young, R. Freedman, University Physics (with Modern Physics), 12, (2008)

4. R.F. Coughlin, F.F. Driscoll, Amplificadores Operacionales y Circuitos Integrado, 5, (2000)

5. R.L. Boylestad, L. Nashelsky, Electrónica: Teoría de Circuitos y Dispositivos Electrónicos, 10, (2009)

\subsection{Composition of light.}

